\title{
Case Report: Melioidosis in non-endemic settings: a case with atypical presentation and diagnostic challenges.
}

\author{
J Veater, FH Lim, N Perera
}

\section{Introduction}

Burkholderiapseudomallei infection is rare in Europe and usually seen in the context of travelrelated infection.

\section{Case report}

We report a case of a 43-year-old male from Gujarat, India living in the UK, who developed relapsed $B$. pseudomallei infection on a background of poorly controlled diabetes, alcoholism and malnutrition. He presented in diabetic ketoacidosis and was admitted to intensive care for multi-organ support.

Admission blood cultures flagged positive after 5-days incubation and an oxidase positive, Gram-negative bacillus grew on blood and chocolate agar after 24 hours. The organism was resistant to gentamicin and colistin but sensitive to ceftazidime, meropenem and piperacillin/tazobactam on disc diffusion testing. It was initially poorly identified as Burkholderiamultivorans and Burkholderiacepacia using MALDI-TOF MS and API 20NE respectively. The organism was sent to the reference laboratory for $B$. pseudomallei PCR which was positive. Urine culture and the dialysis catheter tip subsequently grew Trichosporon asahii.

CT head on admission showed no acute abnormality but CT of the chest/ abdomen/pelvis showed bi-basal cavitatory lung lesions, splenic infarction, and hypodense lesions in both kidneys. CT head repeated 16 days post-admission showed multiple cerebral abscesses. Transthoracic echocardiogram showed no vegetations and sputum was negative for Mycobacterium tuberculosis PCR and culture.

Despite initial response to meropenem, he rapidly deteriorated and developed seizures, likely secondary to new intracerebral abscesses. Positive cultures for $T$. asahii suggest secondary superadded fungal infection. Despite treatment with amphotericin $\mathrm{B}$, isavuconazole and meropenem, the patient died.

\section{Discussion and Conclusion}

This case serves as a reminder that many cases of melioidosis are relapsed infection rather than recently acquired. Immunosuppression is a significant risk factor for relapse. Mortality is high and optimal treatment regimen for severe melioidosis remains unresolved.

Organism identification in a UK laboratory can prove challenging and some MALDI-TOF MS databases fail to identify $B$. pseudomallei adequately. Therefore, vigilance is required to identify suspicious isolates in order to pursue identification by alternative means.

Department of Clinical Microbiology, University Hospitals of Leicester NHS Trust, United Kingdom

Address for correspondence: Dr Nelun Perera, Department of Clinical Microbiology, University Hospitals of Leicester NHS Trust, United Kingdom +441162585002 Email: nelun.perera@uhl-tr.nhs.uk

(D) https://orcid.org/0000-0002-0587-5485 
Melioidosis can occur in patients without a recent history of travel due to relapsing disease. A high level of clinical suspicion is required in order to identify the organism correctly on culture. 